\title{
Impact of Correlated Noises on Additive Dynamical Systems
}

\author{
Chujin $\mathrm{Li}^{1}$ and Jinqiao Duan ${ }^{1,2}$ \\ ${ }^{1}$ School of Mathematics and Statistics, Huazhong University of Science and Technology, Wuhan 430074, China \\ ${ }^{2}$ Department of Applied Mathematics, Illinois Institute of Technology, Chicago, IL 60616, USA
}

Correspondence should be addressed to Chujin Li; lichujin@hust.edu.cn

Received 14 April 2014; Accepted 4 August 2014; Published 3 September 2014

Academic Editor: Wuquan Li

Copyright (C) 2014 C. Li and J. Duan. This is an open access article distributed under the Creative Commons Attribution License, which permits unrestricted use, distribution, and reproduction in any medium, provided the original work is properly cited.

\begin{abstract}
Impact of correlated noises on dynamical systems is investigated by considering Fokker-Planck type equations under the fractional white noise measure, which correspond to stochastic differential equations driven by fractional Brownian motions with the Hurst parameter $H>1 / 2$. Firstly, by constructing the fractional white noise framework, one small noise limit theorem is proved, which provides an estimate for the deviation of random solution orbits from the corresponding deterministic orbits. Secondly, numerical experiments are conducted to examine the probability density evolutions of two special dynamical systems, as the Hurst parameter $H$ varies. Certain behaviors of the probability density functions are observed.
\end{abstract}

\section{Introduction}

Dynamical systems arising from financial, biological, physical, or geophysical sciences are often subject to random influences. These random influences may be modeled by various stochastic processes, such as Brownian motions, Lévy motions, or fractional Brownian motions. A fractional Brownian motion $B_{t}^{H}, t \geq 0$, in a probability space $(\Omega, \mathscr{F}, P)$, with Hurst parameter $H \in(0,1)$, is a continuous-time Gaussian process with mean zero, starting at zero and having the following correlation function:

$$
\mathbb{E}\left[B_{s}^{H} B_{t}^{H}\right]=\frac{1}{2}\left(|t|^{2 H}+|s|^{2 H}-|t-s|^{2 H}\right) .
$$

In particular, when $H=1 / 2$ it is just the standard Brownian motion. The time derivative of a fractional Brownian motion, $d B_{t}^{H} / d t$, as a generalized stochastic process, has nonvanishing correlation $[1,2]$ and it is thus called a correlated noise or colored noise. In the special case of $H=1 / 2$, this noise is uncorrelated and thus is called white noise [3]. Correlated noises appear in the modeling of some geophysical systems [4-6].

For systematic discussions about fractional Brownian motions and their stochastic calculus, we refer to [7-12] and the references therein. Fractional Brownian motions have stationary increments and are Hölder continuous with exponent less than $H$, but they are no longer semimartingales, even no longer Markovian. They possess some other significant properties such as long range dependence and self-similarity which result in wide applications in fields such as hydrology, telecommunications, and mathematical finance. During the last decade or so, several reasonable stochastic integrations with respect to fractional Brownian motions were developed. See, for example, Lin [13], Duncan et al. [14], Decreusefond and Üstunel [15], and the references mentioned therein. Stochastic differential equations (SDEs) driven by fractional Brownian motions also have been attracting more attention recently $[1,10,16-18]$.

In this paper, we consider the following scalar stochastic differential equation (SDE):

$$
d X_{t}=b\left(X_{t}\right) d t+\varepsilon d B_{t}^{H}, \quad X_{0}=x,
$$

where the drift $b(\cdot)$ is a Lipschitz continuous function on $R$, $\varepsilon>0$ is the noise intensity, $B_{t}^{H}$ is a fractional Brownian motion with $H>1 / 2$, and the initial state value $\xi$ is assumed to be independent of the natural filtration of $B_{t}^{H}$. Since this system has a unique solution $[17,19]$, here we intend to understand some impact of correlated noises on this additive dynamical system as the Hurst parameter $H$ varies.

This paper is organized as follows. In Section 2, we set up a fractional white noise analysis framework which makes correlated noises as functionals of standard white noises and 
prove a small noise limit theorem which implies the stochastic continuity of the system with respect to noise intensity. In Section 3, we show that the probability density function of $X_{t}$ satisfies a Fokker-Planck type partial differential equation with respect to the fractional white noise measure. Then, we implement numerical experiments to examine the probability density evolutions as the Hurst parameter $H$ varies. As to one linear system and one double-well system, certain behaviors of the probability density functions are observed.

\section{Analysis Framework and Small Noise Limit}

2.1. Analysis Framework. White noise framework is one natural and flexible stochastic analysis thoughtway, and fractional white noise analysis takes correlated noise as functionals of standard white noise. This approach has shown to be very effective in investigating distributions and path properties of stochastic processes. In the following, we describe the fractional white noise analysis framework.

Let $\mathcal{S}(R)$ be the Schwartz space of rapidly decreasing smooth functions on $R$ and $S^{\prime}(R)$ the space of tempered distributions. And denote by $\langle\cdot, \cdot\rangle$ the dual pairing on $\delta^{\prime}(R) \times$ $\mathcal{S}(R)$. For $1 / 2<H<1$, define

$$
\begin{aligned}
\varphi(s, t) & =H(2 H-1)|s-t|^{2 H-2}, \quad s, t \in R ; \\
c_{H}^{2} & =\frac{H(2 H-1)}{B(H-1 / 2,2-2 H)},
\end{aligned}
$$

where $B(\cdot, \cdot)$ is beta function; $K_{ \pm}(t)=c_{H_{ \pm}} t_{ \pm}^{H-3 / 2}, t_{+}=t \vee 0$, $t_{-}=-(t \wedge 0)$.

Lemma 1. For $f \in \mathcal{S}(R)$, let

$$
\begin{aligned}
& \Gamma_{\varphi} f(u)=\left(K_{-} * f\right)(u)=c_{H} \int_{u}^{\infty}(s-u)^{H-3 / 2} f(s) d s, \\
& \Gamma_{\varphi}^{*} f(t)=\left(K_{+} * f\right)(t)=c_{H} \int_{-\infty}^{t}(t-u)^{H-3 / 2} f(u) d u .
\end{aligned}
$$

Then, for $f, g \in \mathcal{S}(R)$,

$$
\left(\Gamma_{\varphi} f, g\right)_{L^{2}(R)}=\left(f, \Gamma_{\varphi}^{*} g\right)_{L^{2}(R)} ;
$$

that is, $\Gamma_{\varphi}^{*}$ is the dual map of $\Gamma_{\varphi}$.

Now we can only prove the linear map $\Gamma_{\varphi}$ is continuous from $\mathcal{S}(R)$ to $L^{2}(R)$. Since $\Gamma_{\varphi}$ is not continuous from $\mathcal{S}(R)$ to $\mathcal{S}(R)$ (even not a proper operator in $\mathcal{S}(R)$ ), we could not obtain a dual map from $\mathcal{S}^{\prime}(R)$ to $\mathcal{S}^{\prime}(R)$ by duality. By using Itô's regularization theorem, we construct a unique $\mathcal{S}^{\prime}(R)$ valued random variable $T: \mathcal{S}^{\prime}(R) \rightarrow \mathcal{S}^{\prime}(R)$ such that

$$
\langle T \omega, \xi\rangle=\left\langle\omega, \Gamma_{\varphi} \xi\right\rangle \mu-\text { a.e. } \omega,
$$

which extends the map $\Gamma_{\varphi}^{*}$ in view of (5).

Theorem 2. Let $\mu_{\varphi}=\mu \circ T^{-1}$ be the image measure of $\mu$ induced by the map $T$. Then, for any $\xi \in \mathcal{S}(R)$, the distribution of $\langle\cdot, \xi\rangle$ under $\mu_{\varphi}$ is the same as $\left\langle\cdot, \Gamma_{\varphi} \xi\right\rangle$ under $\mu$. In particular,

$$
B_{t}^{H} \equiv\left\langle\omega, \Gamma_{\varphi} \mathbf{1}_{[0, t]}\right\rangle, \quad t \geq 0
$$

is a fractional Brownian motion with Hurst constant $H$. Moreover,

$$
B_{t}^{H}=c_{H}\left(H-\frac{1}{2}\right)^{-1} \int_{-\infty}^{t}\left[(t-u)^{H-1 / 2}-u_{-}^{H-1 / 2}\right] d B_{u},
$$

where $B_{t}(\omega) \equiv\left\langle\omega, \mathbf{1}_{[0, t]}\right\rangle$ is the standard Brownian motion. (See proof in [20].)

Let $\left\{\mathscr{F}_{t}, t \in R_{+}\right\}$and $\left\{\mathscr{F}_{t}^{H}, t \in R_{+}\right\}$be the filtrations generated by $\left\{B_{t}\right\}$ and $\left\{B_{t}^{H}\right\}$, respectively. Then, in view of (8), we have

(1) $\mathscr{F}_{t} \supset T^{-1}\left(\mathscr{F}_{t}^{H}\right)$, for all $t \in R_{+}$;

(2) for any $f \in L^{\infty}\left(\mu_{\varphi}\right), \mathbb{E}_{\mu}\left[T_{*} f \mid \mathscr{F}_{t}\right]=T_{*} \mathbb{E}_{\mu_{\varphi}}[f \mid$ $\left.\mathscr{F}_{t}^{H}\right]$ a.s. $[\mu]$, where $\left(T_{*} f\right)(\omega):=f(T \omega)$. So, the filtrated probability space $\left(\mathcal{S}^{\prime}(R), \mathscr{F}_{t}, \mu\right)$ is the extension of $\left(\mathcal{S}^{\prime}(R), \mathscr{F}_{t}^{H}, \mu_{\varphi}\right)$. Thus the stochastic analysis with respect to measure $\mu_{\varphi}$ could be reduced to the standard white noise framework naturally. Therefore, we choose the standard white noise measure $\mu$ as the reference measure rather than $\mu_{\varphi}$, and this treatment is more useful and more convenient for applications. For more details, we refer to [20] and the reference therein.

2.2. Small Noise Limit. Now, we consider the SDE (2) in fractional white noise framework

$$
d X_{t}=b\left(X_{t}\right) d t+\varepsilon d B_{t}^{H}, \quad X_{0}=x
$$

And to investigate the impact of noise on deterministic dynamical system

$$
\frac{d}{d t} x(t)=b(x(t)), \quad x(0)=x
$$

which is solvable on any finite time interval $[0, T]$. We have the following result.

Theorem 3. The solution $X_{t}$ of (2) converges in probability to the solution $x(t)$ of (10) uniformly on any finite time interval $[0, T]$.

Proof. Firstly, we rewrite the equation as

$$
X_{t}-x(t)=\int_{0}^{t}\left[b\left(X_{s}\right)-b(x(s))\right] d s+\varepsilon B_{t}^{H}
$$

Then, by assuming the Lipschitz condition on $b(x)$ with Lipschitz constant $K>0$, it follows from the Gronwall inequality that

$$
\sup _{0 \leq t \leq T}\left|X_{t}-x(t)\right| \leq \varepsilon e^{K T} \sup _{0 \leq t \leq T}\left|B_{t}^{H}\right| .
$$




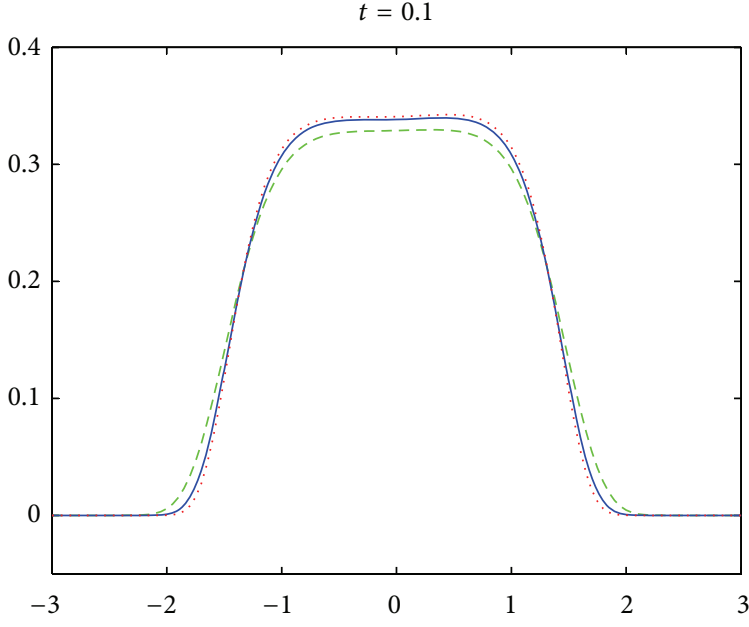

(a)

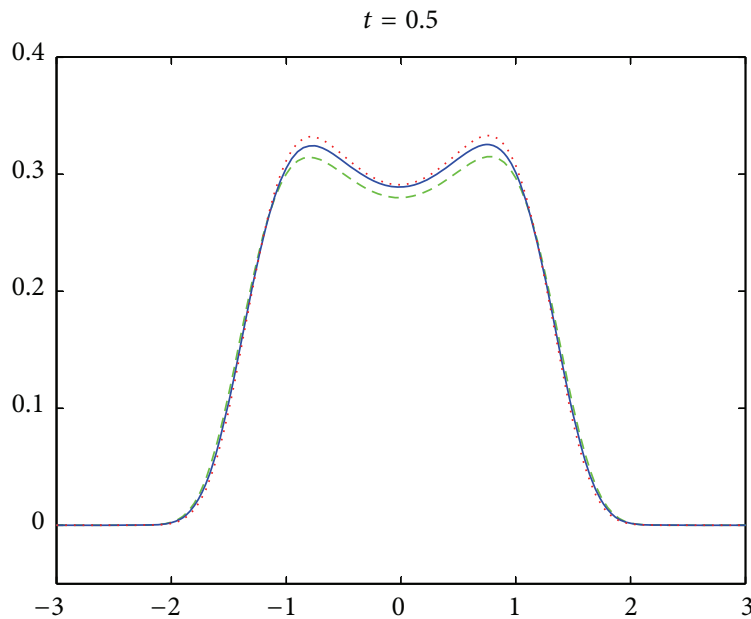

$$
\begin{array}{ll}
\cdots & H=0.5 \\
- & H=0.75 \\
\cdots \cdots & H=0.9
\end{array}
$$

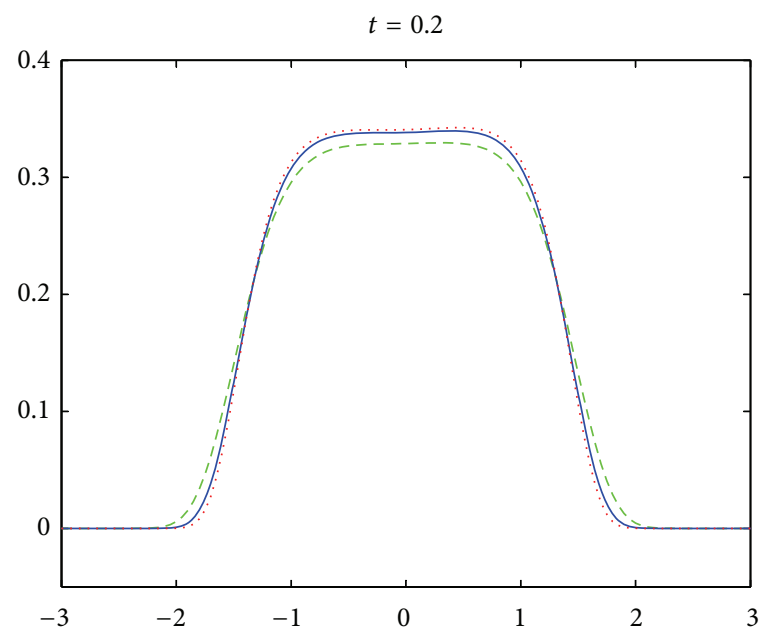

(b)

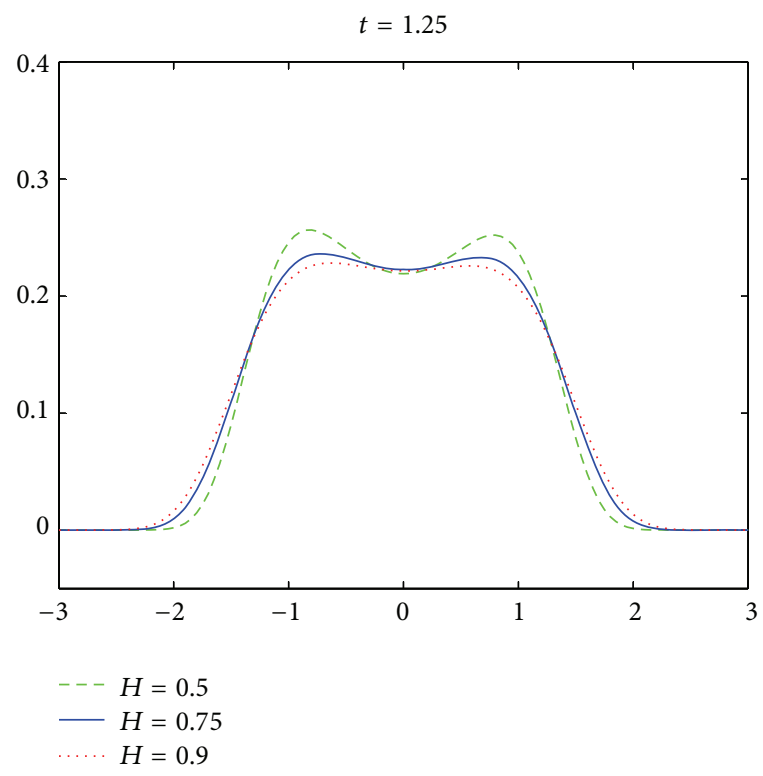

(d)

(c)

Figure 1: Plot of $p(x, t)$ with $b(x)=x-x^{3}$, at $t=0.1,0.2,0.5,1.25$.

Hence, for any small enough $\delta>0$, we have

$$
\begin{aligned}
P\left\{\sup _{0 \leq t \leq T}\left|X_{t}-x(t)\right|>\delta\right\} & \leq P\left\{\sup _{0 \leq t \leq T}\left|B_{t}^{H}\right|>\frac{\delta}{\varepsilon} e^{-K T}\right\} \\
& \leq \frac{\varepsilon e^{K T}}{\delta} \mathbb{E} \sup _{0 \leq t \leq T}\left|B_{t}^{H}\right| \\
& \leq \frac{\varepsilon e^{K T} T^{H}}{\delta} \mathbb{E}\left|B_{1}^{H}\right|,
\end{aligned}
$$

which completes the proof when $\varepsilon \rightarrow 0$. In the final step, we have used the self-similarity of the fractional Brownian motion

$$
\mathbb{E} \sup _{0 \leq t \leq T}\left|B_{t}^{H}\right| \leq T^{H} \mathbb{E}\left|B_{1}^{H}\right|
$$

This theorem provides an estimate for the deviation of random solution orbits from the corresponding deterministic orbits. Note that the expectation $\mathbb{E}$ in the above theorem corresponds to the fractional white noise measure. And, henceforth, we take all expectations $\mathbb{E}$ with respect to the fractional white noise measure (i.e., for simplicity, we omit the subscript $\mu$ mentioned above).

\section{Probability Density Evolution}

For SDE, such as (2), the probability density function of the solution $X_{t}$ carries significant dynamical information. This is considered here by examining a fractional Fokker-Planck type equation. The key step in the derivation of this FokkerPlanck type equation is the application of Ito's formula for SDEs driven by fractional Brownian motion, under fractional 


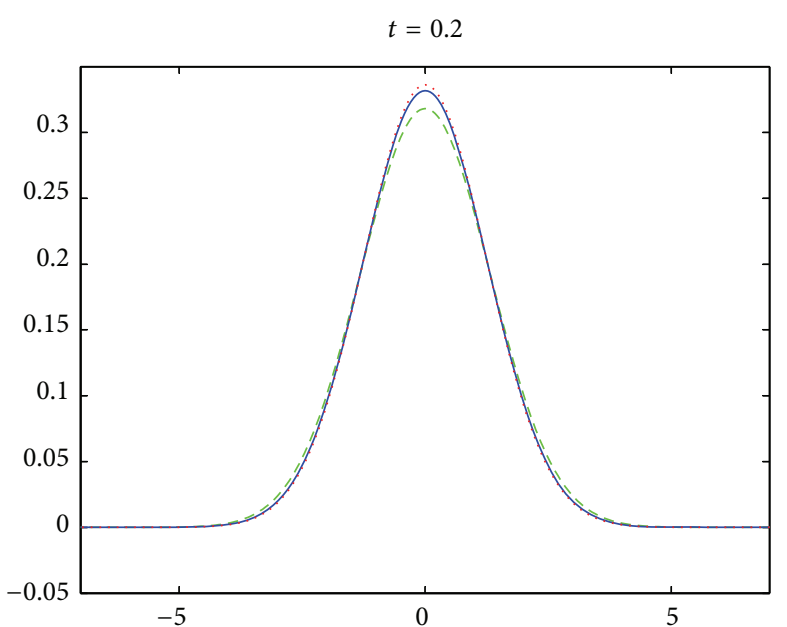

(a)

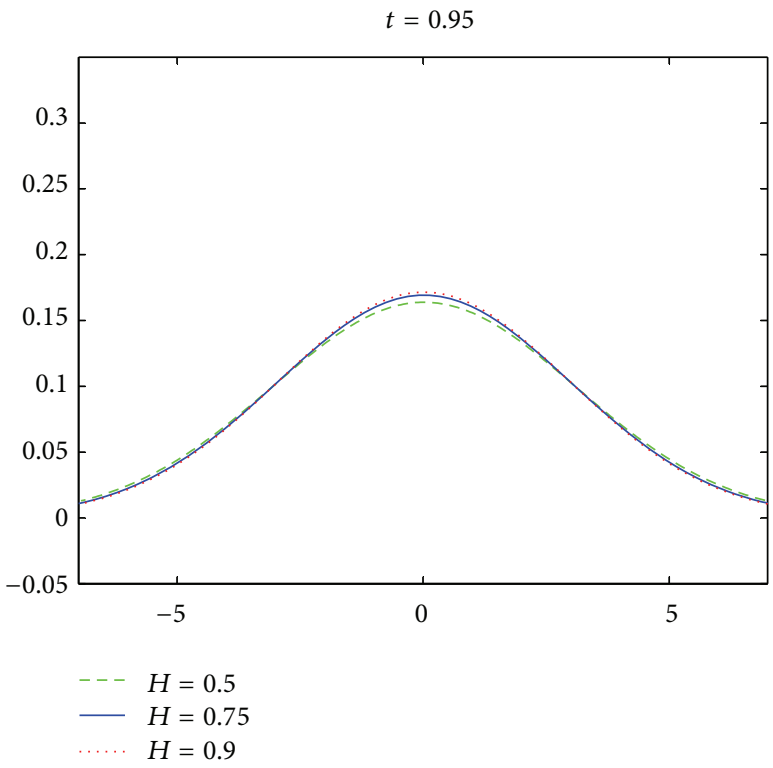

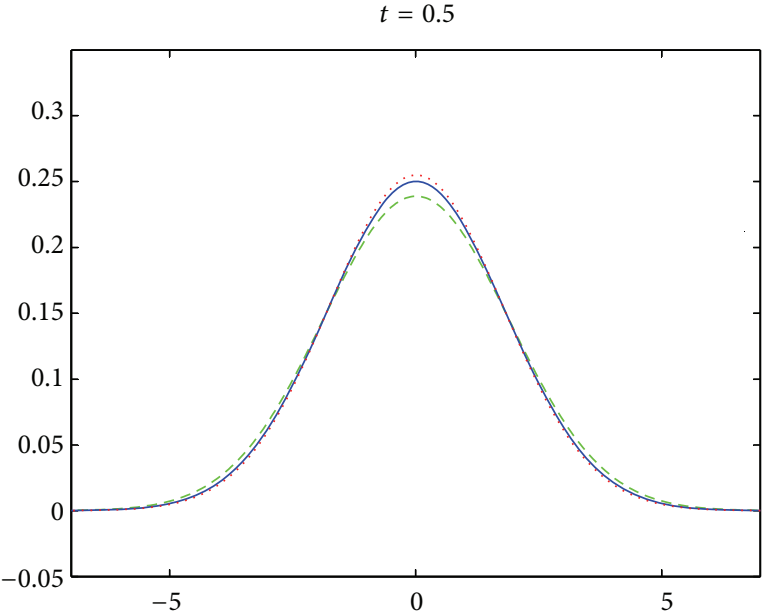

(b)

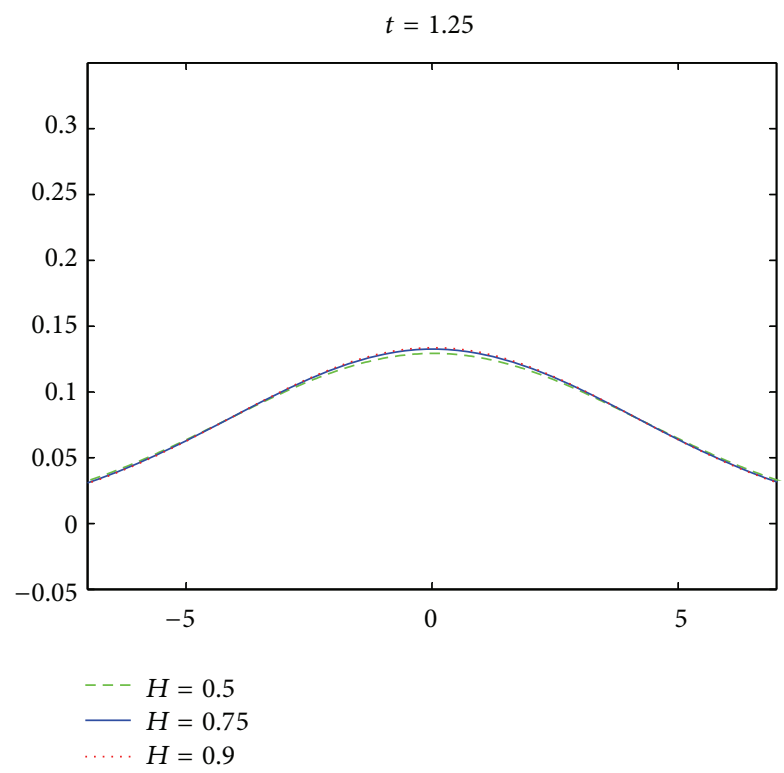

(d)

FIGURE 2: Plot of $p(x, t)$ with $b(x)=x: t=0.2, t=0.5, t=0.95$, and $t=1.25$.

white noise analysis framework $[1,10,16,20,21]$. We sketch the derivation here.

By Ito's formula [10], Theorem 6.3.6, for a second order differentiable function $h(\cdot)$ with compact support, we have

$$
\begin{aligned}
d h\left(X_{t}\right)= & {\left[b\left(X_{t}\right) \frac{\partial h}{\partial x}\left(X_{t}\right)+H t^{2 H-1} \varepsilon^{2} \frac{\partial^{2} h}{\partial x^{2}}\left(X_{t}\right)\right] d t } \\
& +\varepsilon \frac{\partial h}{\partial x}\left(X_{t}\right) d B_{t}^{H} .
\end{aligned}
$$

Taking expectations on both sides yields

$$
\begin{aligned}
\mathbb{E}\left[\frac{d h\left(X_{t}\right)}{d t}\right]= & \mathbb{E}\left[b\left(X_{t}\right) \frac{\partial h}{\partial x}\left(X_{t}\right)\right] \\
& +H t^{2 H-1} \varepsilon^{2} \mathbb{E}\left[\frac{\partial^{2} h}{\partial x^{2}}\left(X_{t}\right)\right] .
\end{aligned}
$$

Let $p=p(x, t)$ be the probability density function of the solution $X_{t}$ of the system (2). Recall that $\mathbb{E}\left[h\left(X_{t}\right)\right]=$ $\int_{\mathbb{R}} h(x) p(x, t) d x$; by integration by parts and $p=0$ at $x=$ $\pm \infty$, we obtain

$$
\int_{\mathbb{R}} h(x)\left[\frac{\partial p}{\partial t}+\frac{b(x) p}{\partial x}-\varepsilon^{2} H t^{2 H-1} \frac{\partial^{2} p}{\partial x^{2}}\right] d x=0 ;
$$

that is,

$$
\frac{\partial p(x, t)}{\partial t}=-\frac{\partial[b(x) p(x, t)]}{\partial x}+\varepsilon^{2} H t^{2 H-1} \frac{\partial^{2} p(x, t)}{\partial x^{2}} .
$$

In the following, we numerically simulate this partial differential equation for two special cases: $b(x)=x-x^{3}$ and $b(x)=x$, with finite noise intensity (for simplicity we take $\varepsilon=1)$. Through these two special cases, we expect to illustrate 
the impact of correlated noises on additive dynamical systems as the Hurst parameter $H$ varies.

Here, we perform the popular Crank-Nicolson scheme in Matlab for (17) with zero boundary values; the grid size is 0.05 , total grid points are 801 , and the time step size is 0.01 . And the initial probability density function is taken to be standard normal; that is, $p(x, 0)=(1 / \sqrt{2 \pi}) e^{-x^{2} / 2}$.

Since the system is tridiagonal, we could solve it using Thomas Algorithm efficiently. Moreover, for other initial conditions and other drift coefficients, for instance, the initial uniform distribution or $b(x)=x-x^{2}$, this method also applies smoothly.

3.1. Numerical Simulation: $b(x)=x-x^{3}$. We first simulate the dynamical evolutions of the probability density function $p(x, t)$ for the corresponding stochastic differential equation (2) with the double-well drift $b(x)=x-x^{3}$, for various values of $H>1 / 2$. The double-well dynamics is a rich and typical model for understanding numerous physical or geophysical systems $[22,23]$, focusing on the maxima (minima), symmetry, kurtosis, and so forth.

As observed in Figure 1, the probability density function $p(x, t)$ evolves from the unimodal (one peak) to the flat top and then to the bimodal (two peaks) shape for various Hurst parameter values $H$, as time $t$ increases. Simultaneously, the effect of Hurst parameter $H$ on the dynamics is significant. As $H$ value increases, the plateau for $p(x, t)$ becomes lower when time exceeds $t=0.5$.

3.2. Numerical Simulation: $b(x)=x$. Now, for comparison we investigate the dynamical evolutions of the probability density function $p(x, t)$ of the corresponding stochastic differential equation (2) with the linear drift $b(x)=x$, which is a rich toy example for understanding dynamical systems.

Also as observed in Figure 2, at given time instants, $p(x, t)$ 's peak becomes higher as $H$ increases. This illustrates the significant and distinguishing influence of Hurst parameter $H$ on the dynamics when time $t$ evolves. The bigger $H$ makes the solution $X_{t}$ of (2) has more centralized value, but the long time effect shows that the values of the solution $X_{t}$ distribute more scatteredly.

\section{Conflict of Interests}

The authors declare that there is no conflict of interests regarding the publication of this paper.

\section{Acknowledgment}

Chujin Li acknowledges the support of Self-Renovation Project HUST2013QN171.

\section{References}

[1] C. Bender, "An Itô formula for generalized functionals of a fractional Brownian motion with arbitrary Hurst parameter," Stochastic Processes and their Applications, vol. 104, no. 1, pp. 81106, 2003.
[2] J. Duan, C. Li, and X. Wang, "Modeling colored noise by fractional Brownian motion," Interdisciplinary Mathematical Sciences, vol. 8, pp. 119-130, 2009.

[3] L. Arnold, Stochastic Differential Equations, John Wiley \& Sons, New York, NY, USA, 1974.

[4] A. Du and J. Duan, "A stochastic approach for parameterizing unresolved scales in a system with memory," Journal of Algorithms \& Computational Technology, vol. 3, no. 3, pp. 393-405, 2009.

[5] J. Duan, "Stochastic modeling of unresolved scales in complex systems," Frontiers of Mathematics in China, vol. 4, no. 3, pp. 425-436, 2009.

[6] B. Chen and J. Duan, "Stochastic quantification of missing mechanisms in dynamical systems," Interdisciplinary Mathematical Sciences, vol. 8, pp. 67-76, 2009.

[7] D. Nualart, "Stochastic calculus with respect to the fractional Brownian motion and applications," Contemporary Mathematics, vol. 336, pp. 3-39, 2003.

[8] E. Alòs and D. Nualart, "Stochastic integration with respect to the fractional Brownian motion," Stochastics and Stochastics Reports, vol. 75, no. 3, pp. 129-152, 2003.

[9] A. Chronopoulou and F. Viens, "Hurst index estimation for selfsimilar processes with long-memory," Interdisciplinary Mathematical Sciences, vol. 8, pp. 91-118, 2009.

[10] F. Biagini, Y. Hu, B. Øksendal, and T. Zhang, Stochastic Calculus for Fractional Brownian Motion and Applications, Springer, New York, NY, USA, 2008.

[11] Y. S. Mishura, Stochastic Calculus for Fractional Brownian Motion and Related Processes, Springer, New York, NY, USA, 2008.

[12] C. A. Tudor and F. G. Viens, "Variations and estimators for self-similarity parameters via Malliavin calculus," The Annals of Probability, vol. 37, no. 6, pp. 2093-2134, 2009.

[13] S. J. Lin, "Stochastic analysis of fractional Brownian motions," Stochastics and Stochastics Reports, vol. 55, no. 1-2, pp. 121-140, 1995.

[14] T. E. Duncan, Y. Hu, and B. Pasik-Duncan, "Stochastic calculus for fractional Brownian motion. I. Theory," SIAM Journal on Control and Optimization, vol. 38, no. 2, pp. 582-612, 2000.

[15] L. Decreusefond and A. S. Üstunel, "Stochastic analysis of the fractional Brownian motion," Potential Analysis, vol. 10, no. 2, pp. 177-214, 1999.

[16] F. Baudoin and L. Coutin, "Operators associated with a stochastic differential equation driven by fractional Brownian motions," Stochastic Processes and Their Applications, vol. 117, no. 5, pp. 550-574, 2007.

[17] M. A. Diop and Y. Ouknine, "A linear stochastic differential equation driven by a fractional Brownian motion with Hurst parameter > 1/2," Statistics \& Probability Letters, vol. 81, no. 8, pp. 1013-1020, 2011.

[18] B. Saussereau, "A stability result for stochastic differential equations driven by fractional Brownian motions," International Journal of Stochastic Analysis, vol. 2012, Article ID 281474, 13 pages, 2012.

[19] L. Denis, M. Erraoui, and Y. Ouknine, "Existence and uniqueness for solutions of one dimensional SDE's driven by an additive fractional noise," Stochastics and Stochastics Reports, vol. 76, no. 5, pp. 409-427, 2004.

[20] Z. Huang and C. Li, "On fractional stable processes and sheets: white noise approach," Journal of Mathematical Analysis and Applications, vol. 325, no. 1, pp. 624-635, 2007. 
[21] G. Ünal, "Fokker-Planck-Kolmogorov equation for fBM: derivation and analytical solution," in Proceedings of the 12th Regional Conference, pp. 53-60, Islamabad, Pakistan, 2006.

[22] D. Farrelly and J. E. Howard, "Double-well dynamics of two ions in the Paul and Penning traps," Physical Review A, vol. 49, no. 2, pp. 1494-1497, 1994.

[23] E. Kierig, U. Schnorrberger, A. Schietinger, J. Tomkovic, and M. K. Oberthaler, "Single-particle tunneling in strongly driven double-well potentials," Physical Review Letters, vol. 100, no. 19, Article ID 190405, 2008. 


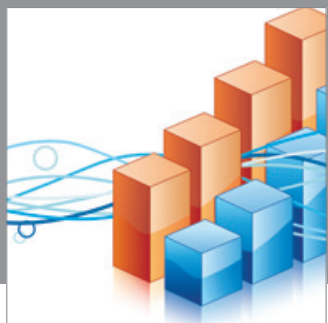

Advances in

Operations Research

mansans

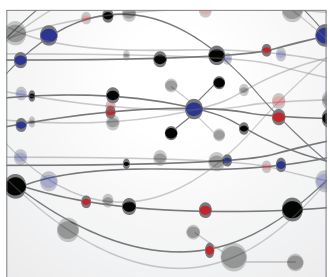

The Scientific World Journal
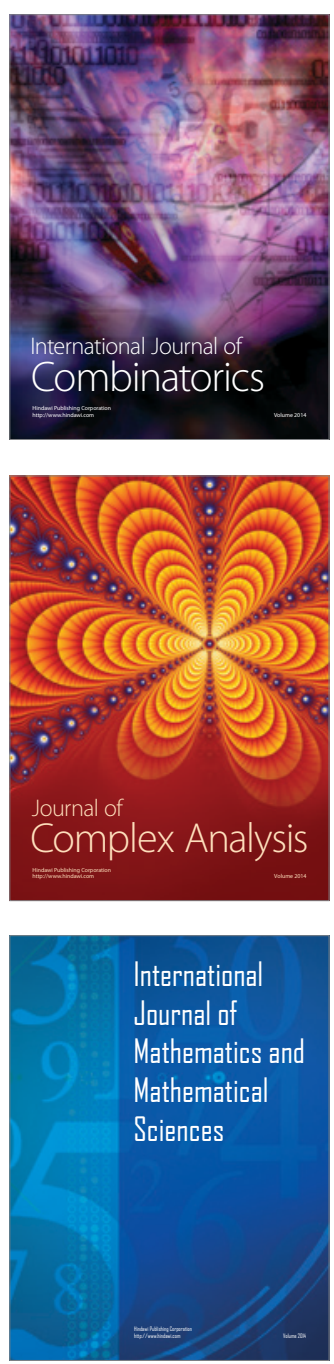
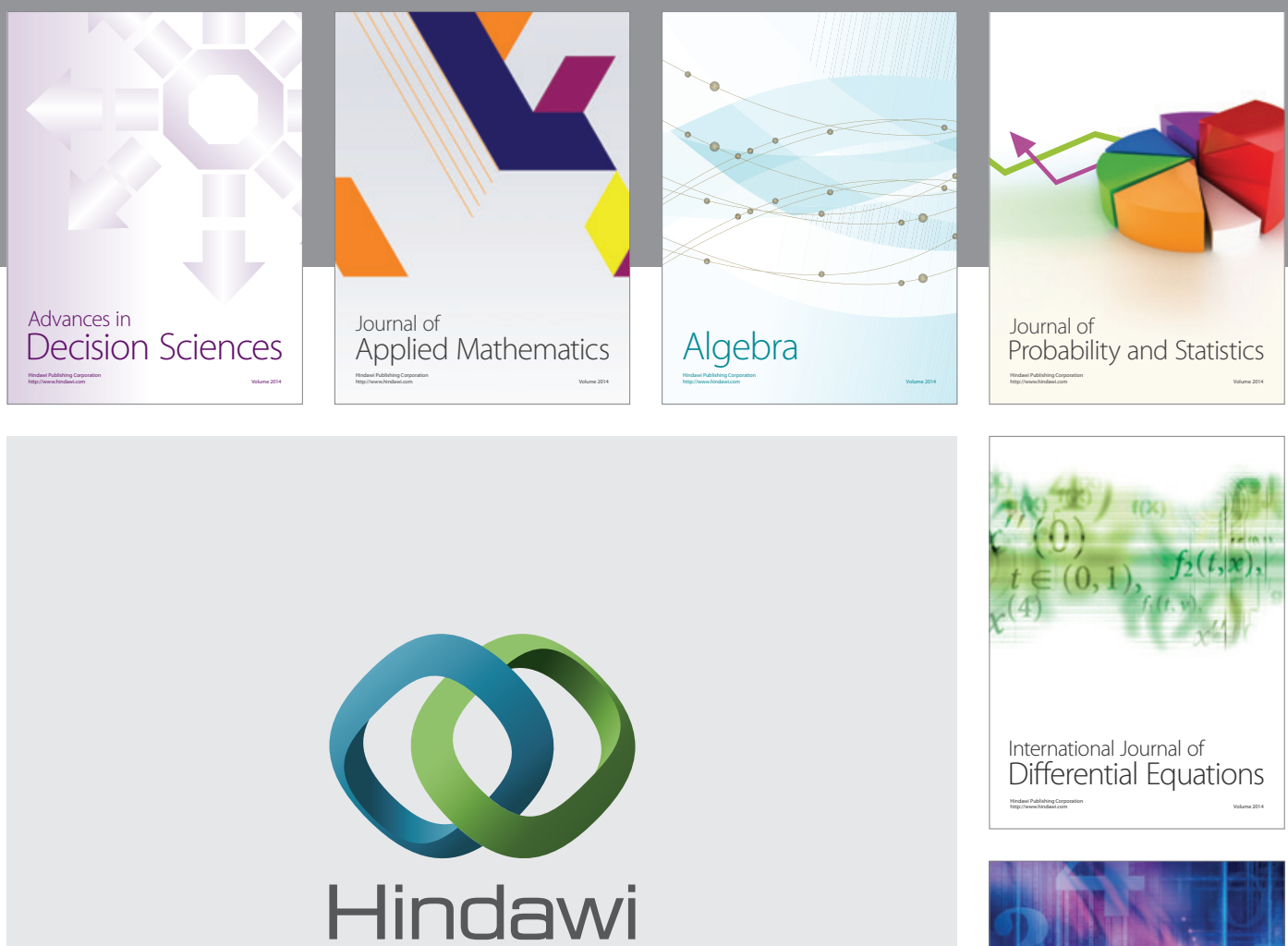

Submit your manuscripts at http://www.hindawi.com
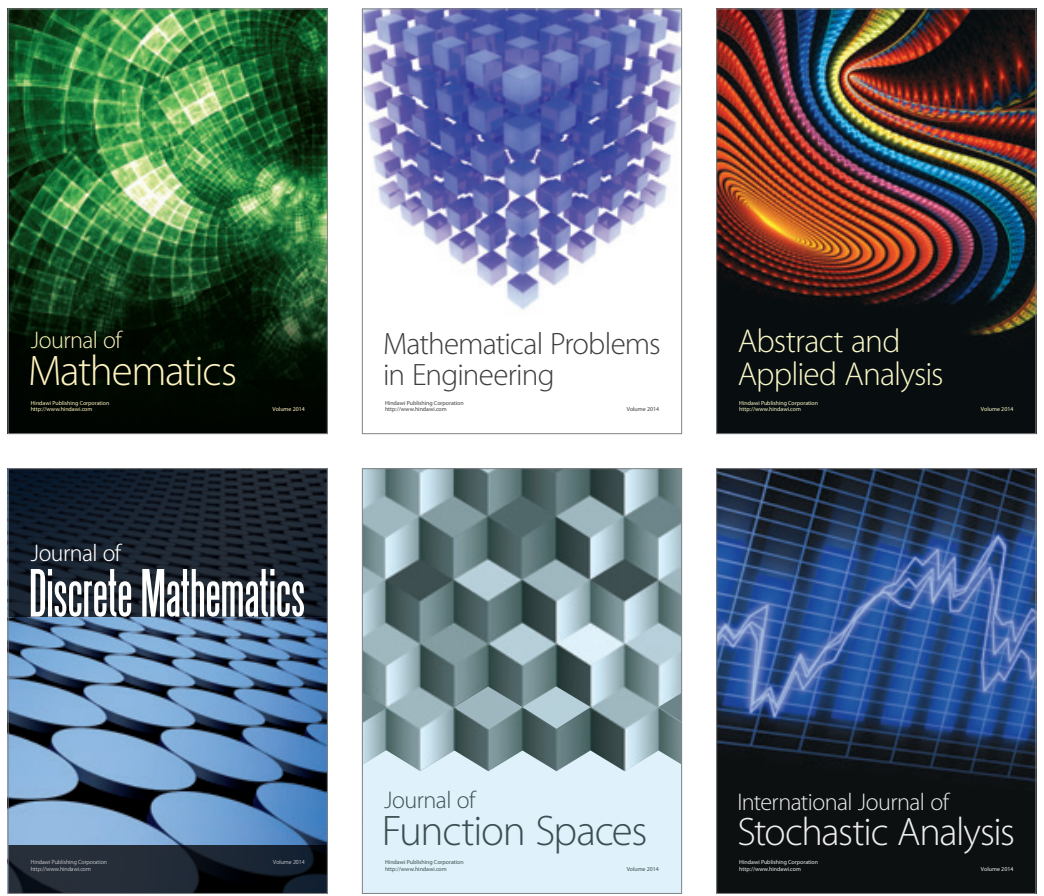

Journal of

Function Spaces

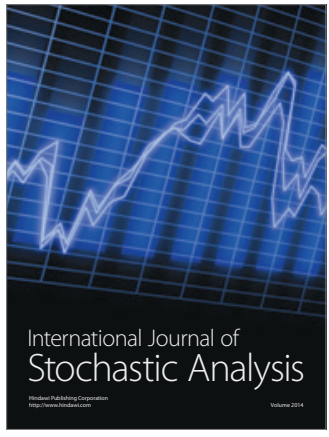

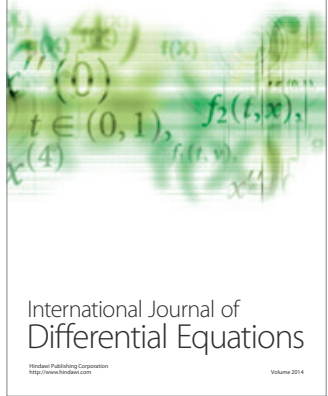
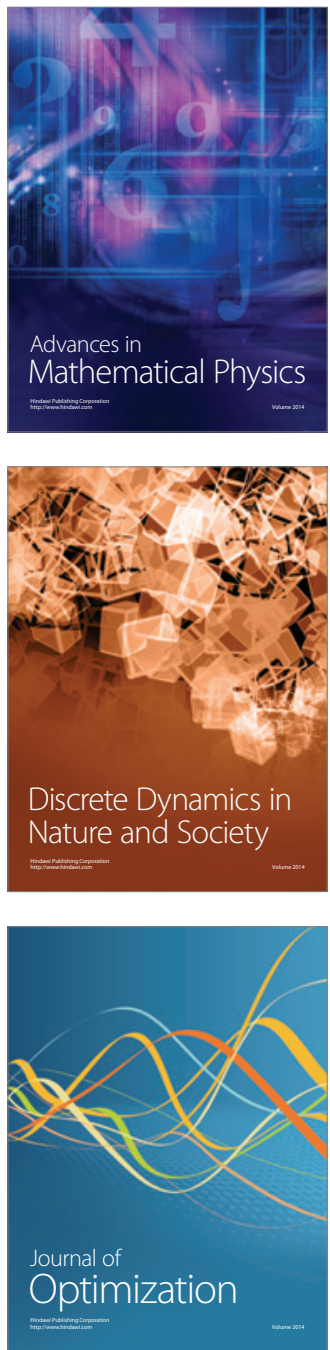